\section{Natural Variances of Mental Tests, and the Symmetry Criterion}

WHes two correlation matrices $R_{1}$ and $R_{2}$ have a symmetrical product $R_{1} R_{2}=R_{2} R_{1}$, they have the same latent vectors ${ }^{1,2}$, and Prof. Cyril Burt has recently proposed ${ }^{3}$ to use this as a criterion that two batteries of mental tests contain the same mental 'factors'. I do not myself think that one can draw any safe conclusions about identity of factors in two batteries unless some tests aro common to both batteries. But if one accepts Burt's criterion, I wish to point out that when $R_{1} R_{2}$ is asymmetrical, the product of the covariance matrices $D_{1} R_{1} D_{1}$ and $D_{2} R_{2} D_{2}$ may conceivably be symmetrical, where $D_{1}$ and $D_{2}$ are diagonal matrices of standard deviations. If an experimenter therefore has psychological reasons for thinking that two batteries contain identical factors, but finds $R_{1} R_{2}$ to be asymmetric, he may be able to discover variances which would make the product $D_{1} R_{1} D_{1} D_{2} R_{2} D_{2}$ symmetric. In that case he would have considerable reason for assuming these to bo the natural variances of these tests.

Godfrex H. Thoyson.

The University, Edinburgh.

August 7.

Eckart, C., and Young, G., Psychometrila, 1, 217 (1936).

Young, G., ibid., 2, 24 (1937).

s Burt, C., ibid., 3, 161 (1938).

\section{Effect of Tube-Length on the Visibility of Dust}

Particles with an Oil-immersion Objective

The phenomenon described by Messrs. Beadle and Patterson in Nature of August 19, p. 327, is well known to practical microscopists, since it occurs whenever a considerable change in the working aperture of a wide-angled objective is made, and admits of a simple explanation.

It is generally admitted that the perfect correction of all the zones of a high aperture oil-immersion objective for spherical aberration is a practical impossibility. It is therefore usual for the computer to concentrate on the inner and outer thirds of the aperture, leaving an intermediate zone in which the corrections are less accurate. When adjusting the tube-length for such an objective under full aperture conditions, the predominating rays for which sym. metry of defocusing is obtained are those from the two preferred zones mentioned above.

In the case of the examination of objects mounted dry upon the cover, and of such a nature that no stray light is reflected or refracted into the portion in excess of N.A. I.0, the first and intermediate zones only are in use, and since the corrections for the rays from the latter will but rarely coincide exactly with those for the outer zone, a change in tube-length will almost certainly be required. The extent of this alteration, and whether plus or minus, will depend entirely on the skill and inclination of the computer, and the figure given by Messrs. Beadle and Patterson of minus $20 \mathrm{~mm}$. to $30 \mathrm{~mm}$. cannot therefore bo regarded as having any scientific significance.

\section{F. E. J. Ockenden.}

59 Stanhope Avenue,

Church End,

Finchley, N.3.

\section{Dipterous Larvæ and Wound Treatment}

Dunng the war of 1914-18, W. S. Baer ${ }^{1}$, in the United States, noticed that when troops wounded in battle had been lying out on the ground unattended for some time, their wounds very commonly became infested with larwe of certain kinds of flies. $\mathrm{He}$ noticed specially that when the wounds had acquired about their maximum maggot population they did not exhibit dangerous after-effects. On the other hand, men whose wounds had received prompt early treatment often developed infection. It was found that the presence of these larvo was especially beneficial in cases of osteomyelitis. It was discovered that the larva were eating the necrotic tissue which was supporting the main bacterial infection, and at the same time these larva were inhibiting further bacterial growth. Stewart ${ }^{2}$ claimed that the scavenging larvi play an important part in clearing the necrotic tissue, but that calcium carbonate, which is constantly excreted by the larva, is also important in alkalizing the medium (the wound) in which the bacteria live, and of increasing phagocytic activity.

The larva used in the earlier treatment of osteo. myelitis belonged chiefly to the species Lucilia sericata (Meig.), L. casar (I.) and Phormia regina (Meig.). Stewart ${ }^{3}$ showed, however, that duo precautions must be taken, and a proper knowledge of the biology of these larva is essential. Some species will feed on both necrotic and normal tissue and are potentially dangerous to the latter.

Much study has been devoted to the technique of rearing dipterous larva under aseptic conditions, and also of retarding their normal development until they were actually needed for implantation within wounds. Sterilization of the eggs in a 5 per cent solution of formaldehyde mixed with a 1 per cent solution of sodium hydroxide for five minutes has been found satisfactory by Robinson". The full details of this process, and that of the subsequent rearing of the larva, is given by the last-named author. Livingston and Prince ${ }^{5}$ were successful in eliminating the inconvenience and unpleasantness associated with the actual 'maggot therapathy' by using a sterile extract of the creatures themselves. Robinson ${ }^{2}$ claimed that the blow-fly larvie contain allantoin in their excretion, and that the rapid healing of wounds as the result of 'maggot thera. pathy', is due in the main to this agent. He did not claim, however, that it could be entirely substituted for the application of the living larva. Robinson carried his investigations further and found that a watery solution of allantoin provides the necessary healing agent, without the presence of dipterous larva at all. In 1935 allantoin was a rare chemical in the laboratory, but it soon came to be made synthetically. Robinson also pointed out that a part of the allantoin moleculo can be split off by hydrolysis to form urea. Further work led to the discovery that a 2 per cent water solution of urea has the equal healing properties of allantoin or of the fly larva themselves. It has the further advantage of being much cheaper than allantoin.

Not only osteomyelitis, but also many other kinds of non-healing wounds, have been treated in the manner just described, including gangrene, heat burns, X-ray burns, varicose and diabetic ulcers, etc. So far as I am aware, Paramonow in $1934^{7}$ was the first to use dipterous larva in the treatment of gas gangrene. He applied maggots of the house fly, Musca domestica, since Calliphora larvæ were prone to devour healthy as well as necrotic tissue. He 